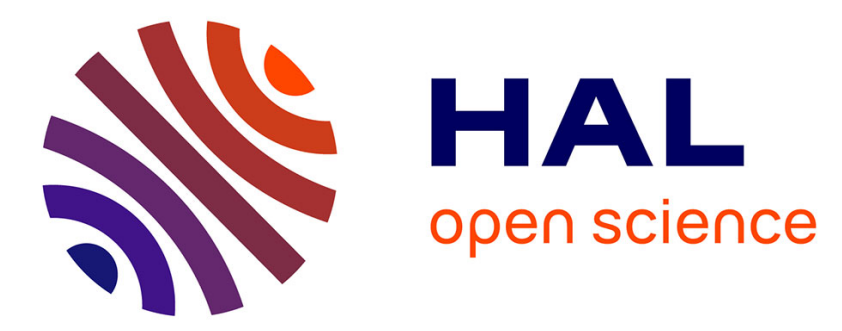

\title{
Synchrotron radiation spectromicroscopy : recent results in neurobiology
}

\author{
G. de Stasio
}

\section{To cite this version:}

G. de Stasio. Synchrotron radiation spectromicroscopy: recent results in neurobiology. Journal de Physique IV Proceedings, 1994, 04 (C9), pp.C9-287-C9-292. 10.1051/.jp4:1994950 . jpa-00253511

\section{HAL Id: jpa-00253511 https://hal.science/jpa-00253511}

Submitted on 1 Jan 1994

HAL is a multi-disciplinary open access archive for the deposit and dissemination of scientific research documents, whether they are published or not. The documents may come from teaching and research institutions in France or abroad, or from public or private research centers.
L'archive ouverte pluridisciplinaire HAL, est destinée au dépôt et à la diffusion de documents scientifiques de niveau recherche, publiés ou non, émanant des établissements d'enseignement et de recherche français ou étrangers, des laboratoires publics ou privés. 


\title{
Synchrotron radiation spectromicroscopy: recent results in neurobiology
}

\author{
G. De Stasio
}

Istituto di Struttura della Materia del CNR, Via Enrico Fermi 38, 00044 Frascati, Roma, Italy and Institut de Physique Appliquée, Ecole Polytechnique Fédérale de Lausanne, 1015 Lausanne, Switzerland

\begin{abstract}
Synchrotron radiation photoemission has recently moved from mere spectroscopy to spectromicroscopy, with lateral resolution that can currently reach the $1000 \AA$ level. This evolution has been so rapid that photoelectron spectromicroscopy experiments are no longer feasibility tests, but real, systematic studies of relevant problems. We present some of the recent results in neurobiology of the XSEM (X-ray Secondary-electron Emission Microscopy) version of this novel approach.
\end{abstract}

\section{Introduction}

Microchemical analysis is an indispensable tool in biology. Synchrotron radiation spectroscopies have had a minimal impact in this field, primarily because of their limited spatial resolution. Recent instrumentation improvements [1-13] made it possible to achieve spatial resolution in synchrotron spectroscopies, giving birth to a new domain called spectromicroscopy. A series of feasibility tests were performed with scanning photoemission spectromicroscopy [6-8,11] and with XSEM,[3-10] i.e., imaging X-ray absorption spectromicroscopy, on biological specimens. Such tests demonstrated the XSEM can be a very effective and flexible tool to investigate the microchemistry of the specimens.

One important point is that the XSEM technique is not highly surface sensitive. It measures [1] the absorption coefficient by detecting [14] the yield of secondary electrons vs the photon energy: since most secondary electrons have low energy, their mean-free-path is not very short, and the probed region's depth is of the order of $50 \AA$. This makes it possible to probe the entire depth of the cell membrane (about $30 \AA$ thick) and a little more -- so that the effects of surface contamination become negligible. Note that biological specimens cannot of course be cleaned with the standard methods of surface science.

The success of the feasibility tests enabled us to initiate a new class of experiments in neurobiology: synchrotron photoelectron spectromicroscopy studies on the distribution of toxic elements in neural specimens.[4,6-10] Toxic elements like aluminum are suspected to be involved in neuropathologies with a dramatic impact on society such as the Alzheimer's and Parkinson's diseases, and related pathologies like the Guam complex.[15-18] Very little is known about the microscopic mechanism of aluminum uptake in the central nervous system. XSEM can significantly contribute to the clarification of issues in this area, since the $\mathrm{x}$-ray absorption edges carry information not only on the presence of the corresponding elements, but also on their chemical status.

The success of this approach will be illustrated with a series of representative examples. Most of them concern brain model systems, i.e., neural cell cultures, which allow manipulations that are impossible for the human brain. This enabled us to observe the physiopathological effects of aluminum in different conditions, for different cells, and for several biochemical pathways.[3-10] The same method has been used for several toxic elements other than aluminum, for example iron, zinc, chromium and cobalt. $[4,10]$ 
Very recently, this approach has been extended for the first time to specimens of human origin.[19] XSEM has detected aluminum in a Guam case brain tissue section, prepared by Perl and his coworkers at the Mount Sinai School of Medicine.

\section{XSEM Results on Neural Cultures}

One of the main advantages of the XSEM technique [1] is that it can provide both microimages and spectra from microscopic areas. It becomes then possible to select the microscopic areas in the image, investigating specific elements and their status in specific portions of the neural structure.
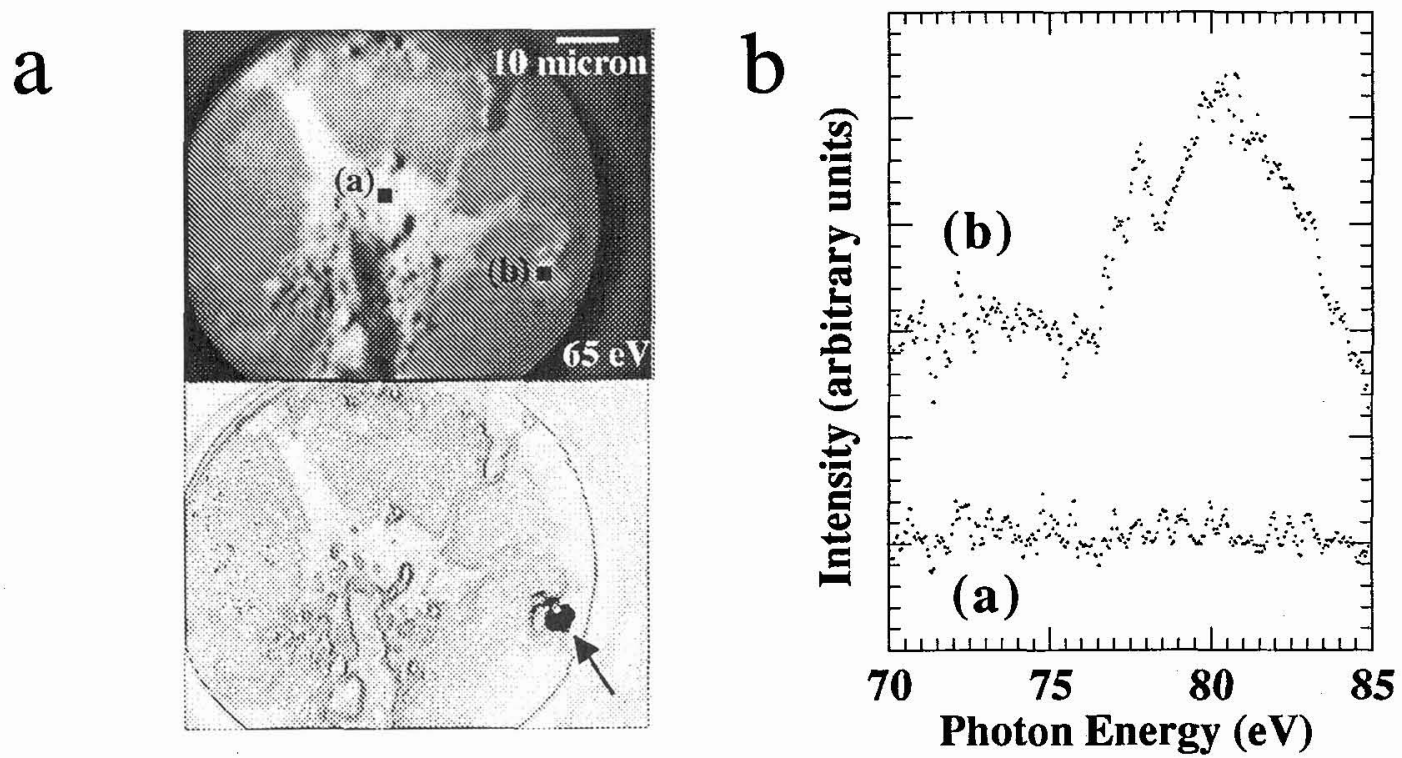

Fig. 1 -- (a) Top: XSEM micrograph of a portion of a rat cerebellar neural culture, that had been exposed to a $5 \mathrm{mM}$ aluminum ion solution for 20 minutes, then carefully washed to remove all of the non-uptaken aluminum, fixed and dehydrated. Bottom: digital subtraction (in black) of two images acquired at 78 and 74 $\mathrm{eV}$ photon energy, above and below the $\mathrm{Al} 2 \mathrm{p}$ absorption edge -- revealing the Al distribution; an intensityattenuated, edge-enhanced version of the top figure is superimposed for reference. The arrow indicates the $\mathrm{Al}$ accumulation region. (b) XSEM spectra taken in the photon energy range of the Al $2 \mathrm{p}$ absorption edge, in the two areas marked in the top part of Fig. 1a. Data from Ref. 9.

Figure 1a shows an example of this approach.[9] The top part of the figure is a micrograph taken with Brian Tonner's XSEM in Wisconsin of a portion of a rat cerebellar neural culture, that had been exposed to aluminum ion solution for 20 minutes, then carefully washed to remove all of the non-uptaken aluminum, fixed and dehydrated. The figure shows a cell, identified on the basis of its morphology as a Purkinje neuron or a glia cell. These are rather rare types of cells for a granule cell culture of this kind: only three were found in the investigated cultures, out of approximately $10^{5}$ cells.

Figure 1b shows XSEM spectra taken in the photon energy range of the $\mathrm{Al} 2 \mathrm{p}$ absorption edge, in the two areas marked in the top part of Fig. 1a. The aluminum edge, revealing the presence of this element. is visible for only one of the two areas.

The spatial distribution of aluminum was then determined by taking two microimages of the same portion of the specimen, at photon energies above and below the edge. The digital subtraction of the two images reveals the aluminum distribution map. This is shown in black in the bottom part of Fig. 1a, superimposed for reference to an intensity-reduced, edge-enhanced version of the micrograph in the top part of the same figure. From results of this type we learn that aluminum is indeed very localized in the culture. More important, aluminum was not found in granule cells, but only in the three aforementioned Purkinje or glia cells. The statistical significance of this result is obvious: a random distribution of aluminum among the cells would have had a probability of only $10^{-15}$ to localize this element in the only three non-granule cells of the cultures. 
The data suggest, therefore, a special role of Purkinje neurons and/or glia cells in the uptake of aluminum. We recently tested [20] this intriguing hypothesis by a second series of tests. Delio Mercanti and Maria Teresa Ciotti of the Istituto di Neurobiologia of the CNR in Rome, Italy, had developed an innovative method to produce cultures that predominantly contain Purkinje neurons.[21] We exposed three of these cultures to aluminum ions in solution, and found by XSEM a statistically much larger cells with aluminum than in the previous studies.

An example of these results [20] is shown in Fig. 2. Figure 2a shows an XSEM microimage of two Purkinje neurons of one of the Mercanti-Ciotti cultures.[21] Figure $2 \mathrm{~b}$ shows aluminum-edge XSEM spectra taken in the marked areas of Fig. 2a. The presence of aluminum in several of these areas is evident. Overall, we explored eight cells in the Mercanti-Ciotti cultures, finding aluminum in six of them. Furthermore, the two cells without aluminum did not have a morphology consistent with that of Purkinje neurons, and were probably spurious cells in the majority Purkinje culture.

Similar results were found for cultures with a predominant content of glia cells. Once again, the statistical occurrence of aluminum uptake is way up with respect to that of the granule-cell cultures.

These findings corroborate the basic conclusion [9] that aluminum uptake preferentially occurs at non-granule cells like Purkinje neurons or glias. This result could have far-reaching consequences in the research on neuropathologies like the Alzheimer's and Parkinson's diseases.
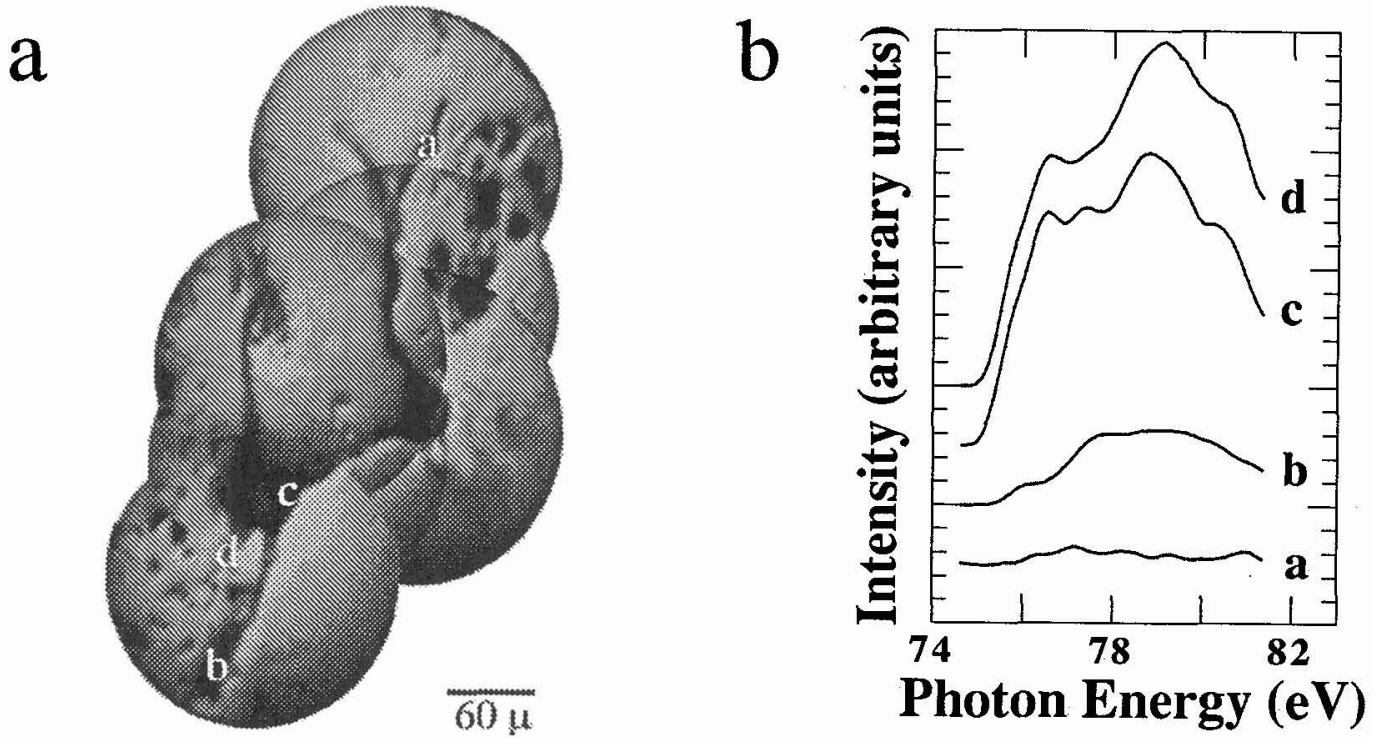

Fig. 2 -- (a) XSEM microimage from a Purkinje neuron culture. (b) Aluminum-edge XSEM spectra taken in the marked areas of Fig. 2a. Unpublished data from De Stasio et al., Ref. 20.

\section{First Tests on Human Brain Tissue}

In the long run, the success of this approach will critically depend on the possibility to extend it beyond cultures and to tissue sections. We present here the first successful feasibility test of this extension: the use of XSEM to detect aluminum in tissue sections from a Guamian patient's brain.[19]

The Guam syndrome always consists [18] of a complex of amyotrophic lateral sclerosis and Parkinson dementia (ALS-PD complex), with predominance of one of the two diseases. It has been linked to the island of Guam, but strong evidence exists against a merely genetic etiology. The very extensive studies notwithstanding, the origin of the ALS-PD complex is still unclear. The interest of this problem goes beyond the ALS-PD complex itself, because of its links with other diseases like the Alzheimer's and the Parkinson's which are widespread in North America. 
In 1980, Perl et al.[16] detected for the first time aluminum in Alzheimer cases' brain specimens, strictly localized to the neurofibrillary tangles: two years afterwards, [15] they found similarly localized aluminum in Guam cases specimens. This led to a major re-direction of the research in this field.

In the present tests, we used again Tonner's XSEM [1] at the Wisconsin Synchrotron Radiation Center to search for aluminum in a Guam case brain tissue section. The specimen was taken at the Mount Sinai School of Medicine from a formalin-fixed brain; the Sommer's sector of the hyppocampus was extracted and thin ( $600 \AA$ thick) sections were obtained with an ultramicrotome. The sections were deposited on a copper mesh and transferred to the Wisconsin synchrotron laboratory.

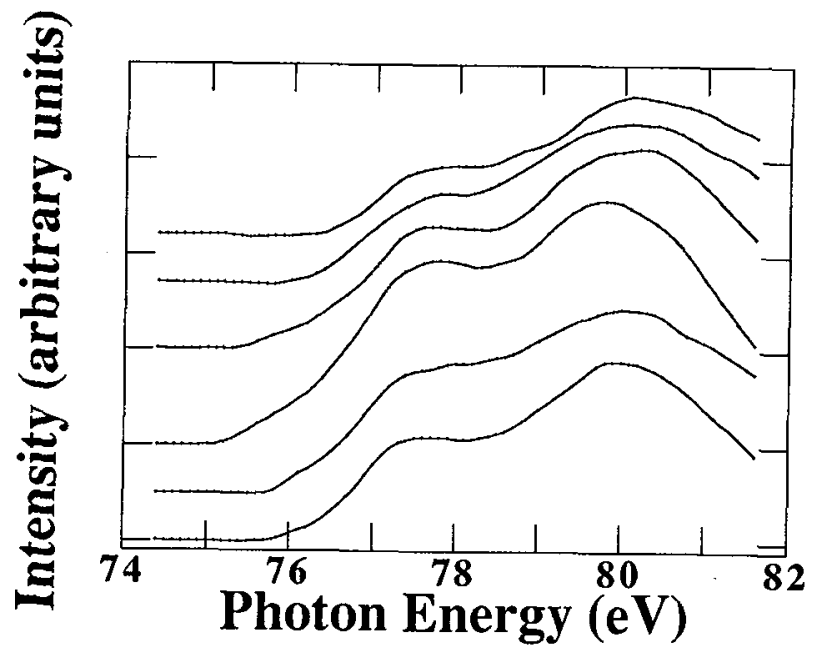

Fig. 3 -- XSEM Al 2 p spectra from $2 \times 2 \mu \mathrm{m}^{2}$ areas of a Guam case brain tissue section specimen, $600 \AA$ thick. Unpublished data from Ref. 19.

Figure 3 shows a representative example of the experimental detection of Al with the XSEM.[19] We see there a series of $\mathrm{Al} 2 \mathrm{p}$ spectra from $2 \times 2 \mu \mathrm{m}^{2}$ areas of the specimen. This result is only a feasibility test, but clearly demonstrates that the XSEM approach is already sensitive enough to detect aluminum in brain sections. The lateral resolution of the technique can possibly reach the level of $100 \AA$ or better, and therefore open new domains in the study of aluminum (and other elements') brain microchemistry. Note that an increase in lateral resolution also corresponds to an increase in the chemical sensitivity (currently limited to 80 $\mathrm{ppm}$ ), because the probed area can better match the localization of the detected element.

\section{Future Outlook}

This is one of the domains that is most likely to profit from the advent of the new third generation of ultrabright synchrotron radiation sources. In fact, microscopy and spectromicroscopy are areas that immediately benefit from the decrease in emittance and subsequent increase in brightness. The Liouville theorem applied to an ideal, lossless beamline, implies the concentration of brightness. This also means that bringing a large number of photons per unit time in a small area of the specimen - as required by most if not all microscopies and spectromicroscopies - is much easier with high brightness.

The past few months brought some outstanding news. Three soft-x-ray high brightness facilities were commissioned in record short times: the Advanced Light Source in Berkeley, the Synchrotron Radiation Research Center source in Taiwan and Elettra in Trieste. These facilities are particularly suited for photoelectron spectromicroscopy.

Quite significantly, the very first test experiment on Elettra [22] included produced photoelectron microscopy ima oes of ne.ural cultures similar tn those discrussed ahove. The historv of the commiscioning nf F.lettra and 
of the first test experiment is one of a remarkable success. The accelerator division, under the guidance of Albin Wrülich, moved in a few days from the first injection attempt to the specification current of $400 \mathrm{~mA}$. Three weeks after the first injection, we obtained the first photoelectron micrographs,[22] including images of neural cultures. These images were obtained with a PEEM (Photo-Electron Emission Microscope), in cooperation with the scientific division of Elettra and with Fabia Gozzo and Tiziana dell'Orto of the Ecole Polytechnique Fédérale de Lausanne. An example is shown in Fig. 4.

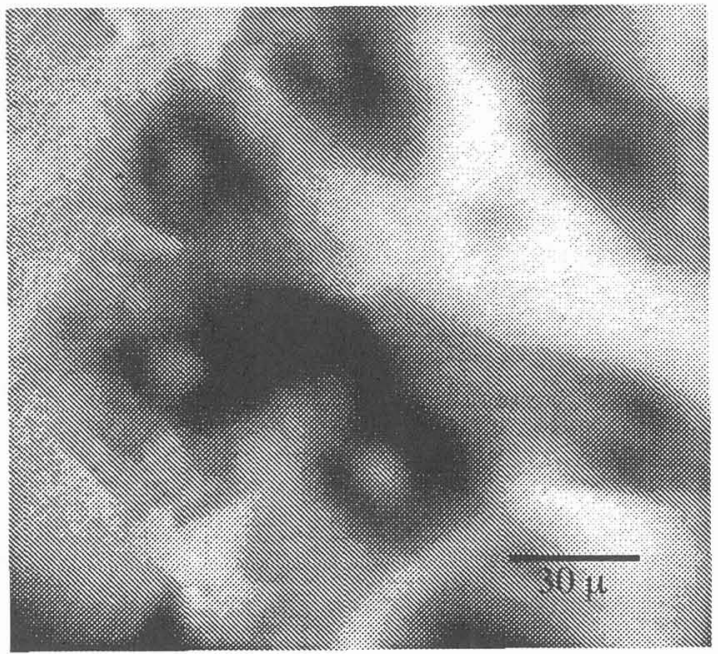

Fig. 4 -- PEEM micrograph from a glia culture, taken on the Elettra synchrotron source in Trieste (unpublished data from Ref. 22). This was part of the first series of test experiments on Elettra (October 27, 1993), only three weeks after the first injection attempt in the storage ring.

This is, of course, just a feasibility test of the new synchrotron facility. But it already demonstrates that Elettra functions well enough to perform spectromicroscopy. Combined with the equally good news of the Taiwan and Berkeley sources, this makes the future of synchrotron spectromicroscopy in biology quite bright if not ultrabright.

\section{Acknowledgments}

Work supported by the Fonds National Suisse de la Recherche Scientifique, by the Consiglio Nazionale delle Ricerche, by the Ecole Polytechnique Fédérale de Lausanne, by the Società Sincrotrone Trieste and by the National Science Foundation, directly and through the Wisconsin Synchrotron Radiation Center, a national facility. A special thank to G. Margaritondo for his constant encouragement and support. I thank the many colleagues with whom I collaborated in this experiments: B. P. Tonner, P. Perfetti, D. Mercanti, M. T. Ciotti, A. Angelini, D. Dunham, T. C. Droubay, Mario Capozi, Bruno Nicolini, Marino Marsi and Tiziana dell'Orto, D. P. Perl, Amy Hsu, Paul Good, F. Gozzo, C. Coluzza, M. Bertolo, A. Wrülich, G. Viani, D. Alfe' and all of the staff members of Elettra.

\section{References}

[1] Tonner B. P. and Harp G. R., Rev. Sci. Instrum 59 (1988) 853-863; Tonner B.P. and Harp G.R., J. Vac. Sci. Technol. 7 (1989); 1-15. Koranda S. F., Zhang J., Rev. Sci. Instrum . 63 (1992) 563569.

[2] Rudolph D., Schmahl G., Niemann B., Guttmann P., Thieme J., Schneider G., David C., Diehl M., Wilhein T., in Vacuum Ultraviolet Radiation Physics, eds. Wuilleumier F. J., Petroff Y. and Nenner I. (World Scientific, Singapore, 1993) pp. 544-556. 
[3] De Stasio G., Koranda S. F., Tonner B. P., Harp G. R., Mercanti D., Ciotti M. T. and Margaritondo G., Europhys. Lett., 19 (1992) 655-659.

[4] De Stasio G., Hardcastle S., Koranda S. F., Tonner B. P., Mercanti D., Ciotti M. T., Perfetti P. and Margaritondo G., Phys. Rev. E 47 (1993) 2117-2121.

[5] De Stasio G., Perfetti P., Oddo N., Galli P., Mercanti D., Ciotti M. T., Koranda S. F., Hardcastle S., Tonner B. P. and Margaritondo G., NeuroReport 3 (1992) 965-968.

[6] De Stasio G., Perfetti P., Mercanti D., Ciotti M. T., Hardcastle S., Koranda S. F., Tonner B. P., Capasso C., Ng. W., Ray-Chaudhury A. K., Liang S., Singh S., Cerrina F. and Margaritondo G., in Vacuum Ultraviolet Radiation Physics, eds. Wuilleumier F. J., Petroff Y. and Nenner I. (World Scientific, Singapore, 1993) pp. 534-543.

[7] De Stasio G., Cerrina F., Tonner B. P., Mercanti D. and Margaritondo G., in Life Chemistry Reports (Harwood, London, 1994) in press.

[8] De Stasio G. and Margaritondo G., in New Directions in Research with Third-Generation Soft X-Ray Synchrotron Radiation Sources, eds. Schlachter A. S. and Wuilleumier F. J. (Kluwer NATO ASI Series, Amsterdam 1994) pp. 299-313.

[9] De Stasio G., Dunham D., Tonner B. P., Mercanti D., Ciotti M. T., Angelini A., Coluzza C., Perfetti P. and Margaritondo G., NeuroReport 4 (1993) 1175-1178.

[10] De Stasio G., Dunham D., Tonner B. P., Mercanti D., Ciotti M. T., Perfetti P. and Margaritondo G., unpublished.

[11] De Stasio G., Perfetti P., Ng. W., Ray-Chaudhury A. K., Liang S., Singh S., Cole R. K., Guo Z. Y., Wallace J., Capasso C., Cerrina F., Mercanti D., Ciotti M. T., Gozzo F. and Margaritondo G., Phys. Rev. E 48 (1993) 1478-1482.

[12] Ade H., Jacobsen C., Kirz J., Ko C.-H., Lindaas S., Williams S., Zhang X., Buckley C., Anderson E., Goncz K. and Rothman S., in Vacuum Ultraviolet Radiation Physics, eds. Wuilleumier F. J., Petroff Y. and Nenner I. (World Scientific, Singapore, 1993) pp. 523-533.

[13] Stohr, J., Wu Y., Hermsmeier, B. D., Samant M. G., Harp G. R., Koranda S., Dunham D., Tonner B. P., Science 259 (1993) 658-662.

[14] Gudat W. and Kunz C., Phys. Rev. Lett. 29 (1972) 169-179.

[15] Perl D. P., Gadjusek D. C., Garruto R. M., Yanagihara R. T. and Gibbs J. Jr., Science 217 (1982) 1053-1055.

[16] Perl D. P. and Brody A. R., Science 208 (1980) 297-301.

[17] Perl D. P. and Good P. F., J. Neuropathol. Exp. Neurol. 47 (1988) 318-322.

[18] Perl D. P., Steele J. C., Loerzel A. and Kurland L. T., in Alzheimer's Disease: Basic Mechanisms, Diagnosis and Therapeutic Strategies, Eds. Iqbal K., McLachlan D. R. C., Winblad B. and Wisniewski H. M. (John Wiley \& Sons Ltd., 1991) pp.375-381.

[19] De Stasio G., Perl D. P., Hsu A., Good P. F., Tonner B. P., Perfetti P. and Margaritondo G., unpublished.

[20] De Stasio G., Dunham D., Droubay T. C., Tonner B. P., Mercanti D., Ciotti M. T., Perfetti P. and Margaritondo G., unpublished.

[21] Mercanti D. and Ciotti M. T., unpublished.

[22] Abrami A., Alfé D., Bertolo M., Capozi M., Ciotti M. T., Coluzza C., De Stasio G., dell'Orto T., Di Fabrizio E., Fontana S., Gentili M., Gozzo F., Margaritondo G., Mazzolini F., Mercanti D., Perfetti P., Rindi G., Rosei R., Savoia A., Viani G., Wrülich A., Rubbia C., unpublished. 